\title{
Electromagnetic Radiation from a Tesla Transformer
}

\author{
R M Craven ${ }^{1}$, I R Smith ${ }^{1} \&$ B M Novac ${ }^{1}$ \\ ${ }^{1}$ Wolfson School of Mechanical, Electrical and Manufacturing Engineering, Loughborough University, \\ Leicestershire LE11 3TU, UK \\ Correspondence: Ivor R Smith, Wolfson School of Mechanical, Electrical and Manufacturing Engineering, \\ Loughborough University, Leicestershire LE11 3TU, UK. E-mail: I.R.Smith@lboro.ac.uk
}

Received: February 10, 2017

Accepted: March 6, 2017

Online Published: March 17, 2017

doi:10.5539/apr.v9n2p53

URL: https://doi.org/10.5539/apr.v9n2p53

\begin{abstract}
In addition to the resistive and dielectric losses that inevitably occur near the secondary winding of a Tesla transformer, electromagnetic radiation into the far field also contributes to the overall power losses and thereby reduces both the effective quality factor $(\mathrm{Q})$ and the power transfer efficiency of this winding. A short study of these effects for a laboratory scale transformer has shown that, in addition to its Q-factor being considerably reduced, the secondary winding is an extremely inefficient radiator of electromagnetic energy.
\end{abstract}

Keywords: Tesla transformers, pulsed power supplies, high-voltage (HV) techniques, electromagnetic radiation

\section{Introduction}

The external loss mechanisms that arise when the fields surrounding the non-isolated secondary winding of a nonsparking Tesla transformer link with elements of the surrounding environment can be quantified as:

- the magnetic field coupling with nearby conducting objects leading to additional resistive losses

- the electric field coupling into nearby dielectric material leading to hysteresis losses

- losses due to the electromagnetic radiation propagating into the far field. Electric field losses are normally negligible in this region

All these losses contribute to both degrading the effective Q-factor and the power transfer efficiency of the winding. They are examined in this paper during the period which begins when all the energy from the primary winding has been transferred to the secondary winding. Current still flows in the resonant system comprising the secondary winding and its topload, such as a sharpening gap, but no current flows from the topload through any other path, such as an ionised air channel or a load.

Although numerous previous papers have been published on Tesla transformers, the great majority of these have been confined to routine performance considerations based on a lumped equivalent circuit. Their continuing importance in technically active countries worldwide is however clearly demonstrated by the many new applications that are still being described, for example (Pecquois, Pecastaing, Rivaletto, de Ferron, \& Vezinet, 2012; Liu et al., 2013; Zhao et al., 2013).

\section{Background}

A relevant definition (Institute of Electronic and Electrical Engineers (IEEE) Standard143-, 1993) of an electrically small antenna (ESA) is "An antenna whose dimensions are such that it can be contained within a sphere whose diameter is small compared to a wavelength at the frequency of operation". Additionally, Wheeler (1976) defines an ESA as having the form of a capacitor or inductor tuned to resonance by an opposite reactance and which can be accommodated within a sphere of radius $r$, where

$$
\mathrm{r}=\lambda / 2 \pi
$$

and $\lambda$ is the wavelength of the fundamental operational frequency of the antenna.

An example of a physically large Tesla transformer demonstrated in Tesla's original Colorado Springs experiments (see Figure 1) employed a secondary winding approximately $2.5 \mathrm{~m}$ in diameter and $3 \mathrm{~m}$ tall, with an operational frequency of $95 \mathrm{kHz}$. The dimensions are clearly of the order of $\lambda / 1000$, and although physically large it was nevertheless an ESA. In fact, for any frequency less than about $1 \mathrm{MHz}$, when the wavelength is $300 \mathrm{~m}$, the secondary winding of a Tesla transformer needs only to be less than about $48 \mathrm{~m}$ long to be regarded as electrically small! 


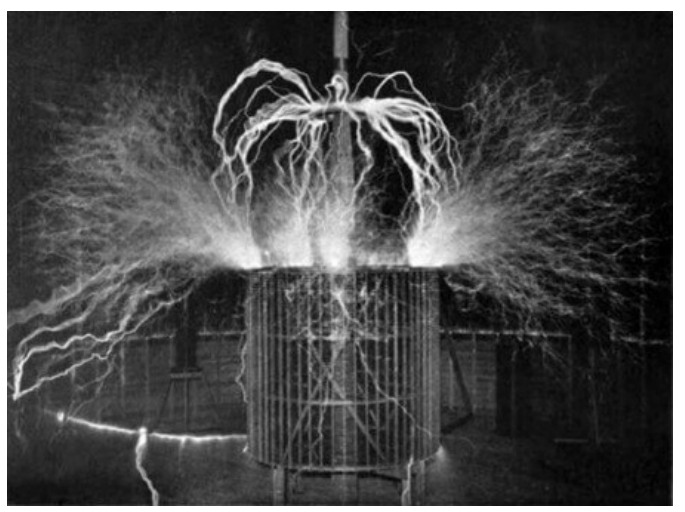

Figure 1. Spectacular Example of Tesla's original experiments at Colorado Springs, USA

Other transformers investigated by the present authors were all found to satisfy the requirements of equation (1), including an experimental unit designed and built at Loughborough University (Craven 2014a). The detailed parameters of this high-voltage transformer that employed only atmospheric pressure air insulation and was used as a reliable and repeatable reference are available elsewhere (Craven, Smith \& Novac, 2017b). The secondary winding had a length of $563 \mathrm{~mm}$, a diameter of $114 \mathrm{~mm}$ and contained 1600 uniformly wound turns of enamelled copper wire in a single-layer solenoid. The magnetic coupling coefficient $\mathrm{k}$ between the primary and secondary windings was 0.145 , and the resonant frequencies of both the primary and secondary circuits in isolation were 136 kHz. Figure 2 presents a view of the experimental Tesla transformer, with the solenoidal secondary winding mounted vertically at the centre of the spiral primary winding.

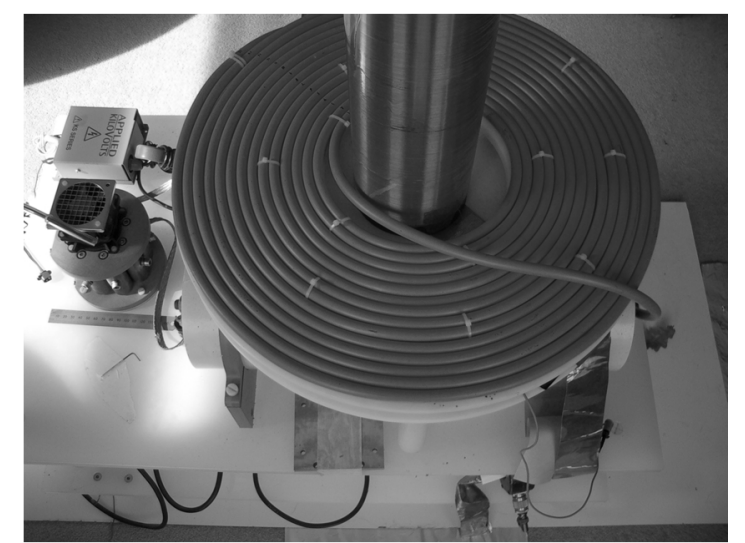

Figure 2. Details of experimental transformer, showing both the vertical solenoidal secondary and the surrounding spiral primary

\subsection{Radial Field Variation.}

The electric and magnetic fields surrounding any antenna are conveniently defined using the boundaries of three distinct regions (Seybold, 2005), in each of which the magnitude of one field component exceeds that of the other two that are present. In order of their distances from the antenna, the three regions are the reactive near field (that extends to a radius of $\lambda / 2 \pi$ ), the radiating near field (the Fresnel region, extending to a radius of $2 D^{2} / \lambda$ ) and the radiating far field (the Fraunhofer region, extending to an infinite radius), where $\lambda$ is the wavelength of the operational frequency and $\mathrm{D}$ is the largest linear dimension of the antenna.

Figure 3 shows the normalised values of the three field components of a typical ESA at its working frequency, as functions of their radial distance from its centre. The figure distinguishes clearly the different ranges at which the different field components are most significant. For the experimental Loughborough transformer, the outer radius of the Fresnel zone of $2.5 \times 10^{-3} \mathrm{~m}$ is much smaller than that of the secondary winding combined with the large radius of curvature of the high-voltage terminal at the output of the secondary winding, This indicates that the radiating near field is contained within the volume occupied by the secondary winding and the high-voltage 
terminal together and that the radiating near field is non-existent, as is evident in Figure 3, where the three field components have equal values at the radius $\lambda / 2 \pi$. In normal operation of the experimental transformer, coupling was observed into objects several metres radially distant, and therefore into the radiating far field.

In addition to the fundamental component, the secondary winding of any Tesla transformer contains harmonic currents at non-integer multiples of the design frequency currents (Craven, 2014a) and the shorter wavelength of these causes the outer boundaries of the radiating near field and the far field of these is extended further outward than those for the fundamental component.

The overall reduction in the Q-factor of the secondary winding of the Loughborough transformer brought about by the combined radiation effects of the external electromagnetic losses resulted in a measured (Craven, Smith, \& Novac, 2014c) value of Q of approximately 200 , a reduction of 50\% from the calculated value when all radiation losses are neglected.

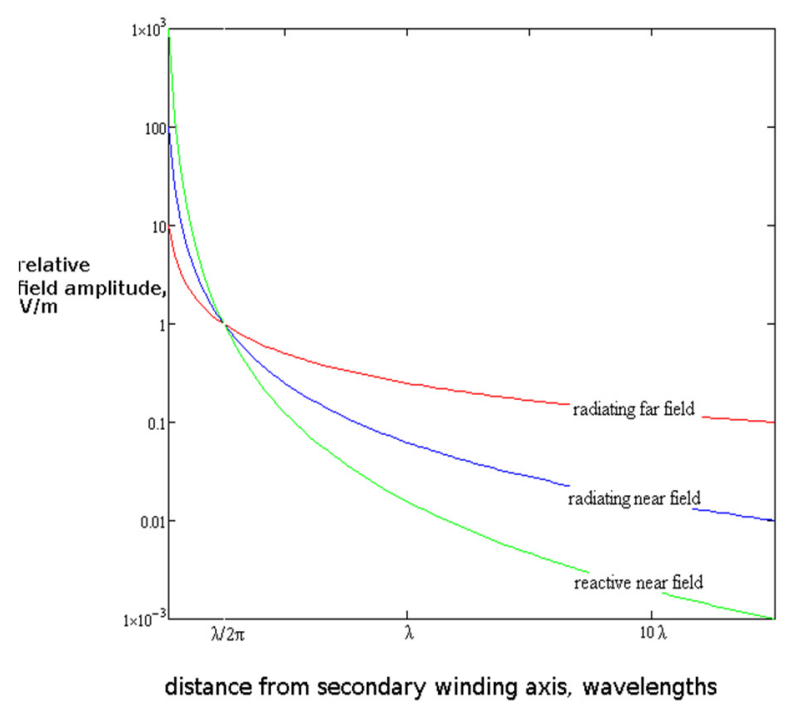

Figure 3 Normalised field strength with increasing distance from secondary winding axis

\section{Radiation Efficiency}

The transfer of energy from the secondary winding into the radiated field can conveniently be represented by that lost in an equivalent radiation resistance $\mathrm{R}_{\mathrm{rad}}$. The conduction and dielectric losses of an ESA are relatively large compared with those in larger Tesla transformers and when they are represented by an equivalent resistance $\mathrm{R}_{\text {loss }}$ in series with $R_{\text {rad }}$ the radiation efficiency $\eta_{\text {rad }}$ may be expressed as

$$
\eta_{\mathrm{rad}}=\mathrm{R}_{\mathrm{rad}} /\left(\mathrm{R}_{\mathrm{rad}}+\mathrm{R}_{\text {loss }}\right)
$$

The various loss mechanisms outlined earlier are often relatively low in large Tesla transformers and frequently insignificant. For efficient energy transfer, the value of $\mathrm{R}_{\text {loss }}$ will therefore be small compared with $\mathrm{R}_{\mathrm{rad}}$ and this is normally true of a Tesla transformer designed to dissipate power in a connected load rather than being lost in EM radiation. The most relevant empirical formulae for calculating the radiation resistance of an ESA (Kandoian \& Sichak, 1953) gave an approximate value of $33 \mu \Omega$ for the Loughborough experimental transformer which is extremely low compared with the RF or even the DC resistances of approximately $200 \Omega$ and $100 \Omega$ respectively, and gave a radiation efficiency of $1.6 \times 10^{-5} \%$.

A typical Tesla transformer secondary winding will have a fundamental resonant frequency of hundreds of $\mathrm{kHz}$, with the odd harmonics that are present reaching up to a few MHz. For a seventh-harmonic frequency of $1 \mathrm{MHz}$, the empirical formula establishes that the radiation resistance still remains at a very low value of approximately 2 $\mathrm{m} \Omega$ Calculations using these figures again gave an extremely low figure for the radiation efficiency, confirming the observed result that the resonant secondary winding of a Tesla transformer is a very poor EM radiator indeed. 


\section{Conclusion}

Although the Q-factor of the secondary winding remains quite high the various local losses due to electromagnetic radiation bring about a significant reduction that must be taken into account in any design optimisation of a Tesla transformer. Furthermore, the calculations have demonstrated that the far field radiation efficiency is extremely low, illustrating convincingly the very desirable feature that a non-sparking Tesla transformer is an extremely inefficient EM radiation source. However, the relative value of $\mathrm{R}_{\text {loss }}$ for transformers larger than the experimental unit is likely to be somewhat lower and the radiation efficiency correspondingly greater, showing that an increased proportion of the energy will be stored in the surrounding far field.

\section{References}

Craven, R. M. (2014a). A study of secondary winding designs for the two-coil Tesla transformer (Doctoral thesis). Loughborough University, Leicestershire LE11 3TU.

Craven, R. M., Smith, I. R., \& Novac, B. M. (2014c). Quality factor measurements of air-cored solenoids at overtone frequencies. Electronics Letters, 50(21), 1528-1529.

Craven, R. M., Smith, I. R., \& Novac, B. M. (2017b). Improvements to Secondary Windings of Tesla Transformers. Applied Physics Research, 9(1), 93-98.

Institute of Electronic and Electrical Engineers. (n.d.). Standard definition of terms for antennas, IEEE Std 1451993.

Kandoian. A., \& Sichak, W. (1953). Wide-frequency-range tuned antennas and circuits. Institution of Radio Engineers International Convention Record, 1, 42-47.

Liu, Y., Lee, L., Bing, Y., Ge, Y., Hu, W., \& Lin, F. (2013). Resonant Charging Performance of Spiral Tesla Transformer Applied in Compact High-Voltage Repetitive Nanosecond Pulse Generator. IEEE Transactions on Plasma Science, 41(12), 3651-3658.

Pecquois, R., Pecastaing, L., Rivaletto, M., de Ferron, A. S., \& Vezine, R. (2012). MOUNA: An Autonomous, Compact, High-Power and Wideband Electromagnetic Source Based on a Novel Resonant Pulsed Transformer. IEEE Transactions on Plasma Science, 40(5), 1407-1415.

Seybold, J. S. (2005). Introduction to RF Propagation. Wiley-Interscience.

Wheeler, H. A. (1976). Small antennas. IEEE Transactions on Antennas and Propagation, 23(4), 139-142.

Zhao, L., Pan, Y. F., Zhang,X.B., Wang, L.M., Fang.J.P., Sun,X. \& Lui. R., (2013). A Tesla-type repetitive pulse generator for solid dielectric research. Review of Scientific Instruments, 84(10), 105-114.

\section{Copyrights}

Copyright for this article is retained by the author(s), with first publication rights granted to the journal.

This is an open-access article distributed under the terms and conditions of the Creative Commons Attribution license (http://creativecommons.org/licenses/by/4.0/). 